\title{
Finite-Time-Singularity with Noise and Damping
}

\author{
Hans C. Fogedby \\ Institute of Physics and Astronomy, University of Aarhus, DK-8000, Aarhus C, Denmark \\ and \\ NORDITA, Blegdamsvej 17, DK-2100, Copenhagen Ø, Denmark
}

Received on 14 May, 2003

\begin{abstract}
The combined influence of linear damping and noise on a dynamical finite-time-singularity model is considered for a single degree of freedom. The noise resolves the finite-time-singularity and replaces it by a first-passagetime distribution with a peak at the singularity and a long time tail. The damping introduces a characteristic cross-over time. In the early time regime the first-passage-time distribution shows a power law behavior with scaling exponent depending on the ratio of the non linear coupling strength to the noise strength. In the late time regime the damping prevails. The study might be of relevance in the context of hydrodynamics on a nanometer scale, in material physics, and in biophysics.
\end{abstract}

\section{Introduction}

The influence of noise on the behavior of nonlinear dynamical system is a recurrent theme in modern statistical physics [1]. In a particular class of systems the nonlinear character gives rise to finite-time-singularities, that is solutions which cease to be valid beyond a particular finite time span. One encounters finite-time-singularities in stellar structure, turbulent flow, and bacterial growth [2, 3, 4]. The phenomenon is also seen in Euler flows and in free-surface-flows [5, 6].

In the context of hydrodynamical flow on a nanoscale [8], where microscopic degrees of freedom come into play, it is a relevant issue how noise influences the hydrodynamical behavior near a finite-time-singularity. Leaving aside the issue of the detailed reduction of the hydrodynamical equations to a nanoscale and the influence of noise on this scale to further study, we assume in the present context that a single variable or "reaction coordinate" effectively captures the interplay between the singularity and the noise.

\section{Model without damping}

In a recent paper [13] we investigated a simple generic model system with one degree of freedom governed by a nonlinear Langevin equation driven by Gaussian white noise,

$$
\frac{d x}{d t}=-\frac{\lambda}{2|x|}+\eta, \quad\langle\eta \eta\rangle(t)=\Delta \delta(t) .
$$

The model is characterized by the coupling parameter $\lambda$, determining the amplitude of the singular term and the noise parameter $\Delta$, determining the strength of the noise correlations. Specifically, in the case of a thermal environment at temperature $T$ the noise strength $\Delta \propto T$.

In the absence of noise this model exhibits a finitetime-singularity at a time $t_{0}$, where the variable $x$ van- ishes with a square law dependence. When noise is added the finite-time-singularity event at $t_{0}$ becomes a statistical event and is conveniently characterized by a first-passagetime distribution $W(t)$ [9]. For vanishing noise we have $W(t)=\delta\left(t-t_{0}\right)$, restating the presence of the finite-timesingularity. In the presence of noise $W(t)$ develops a peak about $t=t_{0}$, vanishes at short times, and acquires a long time tail.

The model in Eq. (1) has also been studied in the context of persistence distributions related to the nonequilibrium critical dynamics of the two-dimensional XY model [10] and in the context of non-Gaussian Markov processes [11]. Finally, regularized for small $x$, the model enters in connection with an analysis of long-range correlated stationary processes [12].

From our analysis in ref. [13] it followed that the distribution at long times is given by the power law behavior

$$
W(t) \sim t^{-\alpha}, \quad \alpha=\frac{3}{2}+\frac{\lambda}{2 \Delta} .
$$

For vanishing nonlinearity, i.e., $\lambda=0$, the finite-timesingularity is absent and the Langevin equation (1) describes a simple random walk of the reaction coordinate, yielding the well-known exponent $\alpha=3 / 2$ [14]. In the nonlinear case with a finite-time-singularity the exponent attains a non-universal correction, depending on the ratio of the nonlinear strength to the strength of the noise; for a thermal environment the correction is proportional to $1 / T$.

\section{Model with damping}

In our studies so far we have ignored damping. It is, however, clear that in realistic physical situations friction or damping must enter on the same footing as the noise. This follows from the Einstein relation or more generally from 
the fluctuation-dissipation theorem relating the damping to the noise. In the present paper we attempt to amend this situation and thus summarize the results of an extension of the analysis in ref. [13] to the case of linear damping.

For this purpose we consider the following model for one degree of freedom:

$$
\frac{d x}{d t}=-\gamma x-\frac{\lambda}{2|x|}+\eta, \quad\langle\eta \eta\rangle=\Delta \delta(t) .
$$

In addition to the coupling parameter $\lambda$, and the noise parameter $\Delta$, this model is also characterized by the damping constant $\gamma$. Assuming for convenience a dimensionless variable $x$ the coupling, noise strength, and damping, $\lambda, \Delta$, and $\gamma$ have the dimension $1 /$ time. The ratios $\lambda / \Delta$ and $\gamma / \Delta$ are thus dimensionless parameters characterizing the behavior of the system. In terms of a free energy or potential $F$ we can express Eq. (3) in the form

$$
\frac{d x}{d t}=-\frac{1}{2} \frac{d F}{d x}+\eta(t)
$$

where $F$ is given by

$$
F=\gamma x^{2}+\lambda \ln |x| .
$$

The free energy has a logarithmic sink and drives $x$ to the absorbing state $x=0$. For large $x$ the free energy has the form of a harmonic well potential confining the motion. In Fig. 1 we have depicted the noiseless solution for $\eta=0$ and the free energy in the various cases.

In order to model an experimental situation the firstpassage-time distribution $W(t)$ is of direct interest. Firstpassage properties in fact underlie a large class of stochastic processes such as diffusion limited growth, neuron dynamics, self-organized criticality, and stochastic resonance [9].
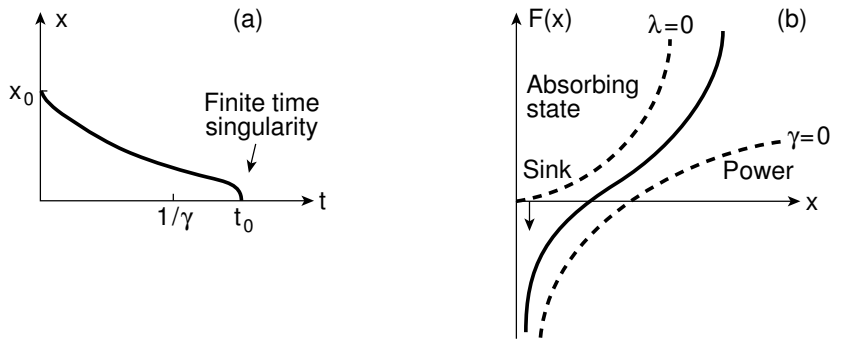

Figure 1. In a) we show the time evolution of the single degree of freedom $x$. At times shorter than the cross-over time $1 / \gamma$ the variable $x$ falls off exponentially. At times beyond $1 / \gamma$ the variable $x$ reaches the absorbing state $x=0$ at a finite time $t_{0}$. In $\mathrm{b}$ ) we depict the free energy $F(x)$ driving the equation. For $\lambda=0$ the free energy forms a confining harmonic well, for $\gamma=0$ we have the absorbing state case discussed in ref. [13]. In the general case the absorbing state $x=0$ corresponds to the sink in $F(x)$.

In term of the distribution function $P(x, t)$ the absorbing state distribution $W(t)$ is defined as $W(t)=$ $-\int_{0}^{\infty} \partial P(x, t) / \partial t d x$. In the absence of noise $P(x, t)=$ $\delta(x-x(t))$ and $W(t)=\delta\left(t-t_{0}\right)$, in accordance with the finite time singularity at $t=t_{0}$. For weak noise we anticipate that $W(t)$ will peak about $t_{0}$ with vanishing tails for small $t$ and large $t$.

In an analysis to be detailed elsewhere we have solved the Fokker-Planck equation associated with the Langevin equation (3),

$$
\frac{\partial P}{\partial t}=\frac{\Delta}{2} \frac{\partial^{2} P}{\partial x^{2}}+\left(\gamma x+\frac{\lambda}{2 x}\right) \frac{\partial P}{\partial x}+\left(\gamma-\frac{\lambda}{2 x^{2}}\right) P
$$

analytically and have found for the probability distribution $P(x, t)$

$$
P(x, t)=\frac{\tilde{x}_{0}^{\lambda / 2 \Delta+1 / 2}}{\tilde{x}^{\lambda / 2 \Delta-1 / 2}} \frac{\gamma e^{\gamma t / 2}}{\Delta \sinh \gamma t} \exp \left[-\frac{\gamma\left(\tilde{x}^{2}+\tilde{x}_{0}^{2}\right)}{2 \Delta \sinh \gamma t}\right] I_{\frac{1}{2}+\frac{\lambda}{2 \Delta}}\left(\frac{\gamma}{\Delta} \frac{\tilde{x} \tilde{x}_{0}}{\sinh \gamma t}\right),
$$

and correspondingly for the first-passage-time distribution $W(t)$

$$
W(t)=\frac{2 \Delta \tilde{x}_{0}^{1+\lambda / \Delta}}{\Gamma(1 / 2+\lambda / 2 \Delta)} \exp \left[-\frac{\gamma \tilde{x}_{0}^{2}}{2 \Delta \sinh \gamma t}\right] \exp (\gamma t)\left(\frac{\gamma}{2 \Delta \sinh \gamma t}\right)^{\frac{3}{2}+\frac{\lambda}{2 \Delta}} .
$$

In Eq. (7) $I_{\nu}$ is the Bessel function of imaginary argument, $I_{\nu}(z)=(-i)^{\nu} J_{\nu}(i z)[15]$ and we have introduced the time scaled variables

$$
\begin{aligned}
& x=\tilde{x} \exp (-\gamma t / 2), \\
& x_{0}=\tilde{x}_{0} \exp (+\gamma t / 2) .
\end{aligned}
$$

From an analysis of Eq. (8) it follows that the damping constant sets an inverse time scale $1 / \gamma$. At intermediate time scales for $\gamma t \ll 1$ the distribution exhibits the same power law behavior as in the undamped case given by Eq. (2). At long times for $\gamma t \gg 1$ the distribution falls off exponentially with time constant $1 / \gamma(1+\lambda / \Delta)$, i.e.,

$$
W(t) \propto \exp [-\gamma(1+\lambda / \Delta) t] .
$$

In the short time limit $W(t)$ vanishes exponentially and shows a maximum about the finite-time-singularity. In Fig. 2 we have depicted the first-passage-time-distribution as a function of t. In Fig. 3 we illustrate the behavior of $W(t)$ in a log-log representation. 


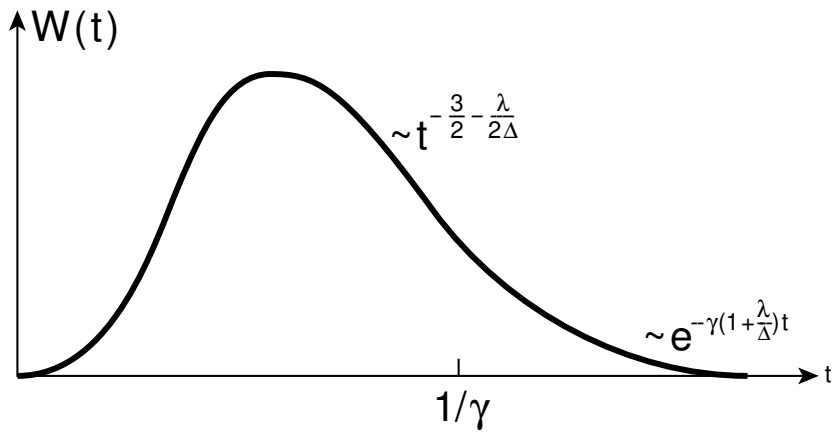

Figure 2. We sketch the first-passage-time distribution $W(t)$ as a function of $t$. In the limit $t \rightarrow 0 W(t)$ vanishes exponentially; about the finite-time-singularity $W(t)$ exhibits a maximum. At intermediate times for $\gamma t \ll 1$ the distribution exhibits a power law behavior with scaling exponent $3 / 2+\lambda / 2 \Delta$. In the long time limit for $\gamma t \gg 1$ an exponential fall-off with time constant $\gamma(1+\lambda / \Delta)$ characterizes the behavior of $W(t)$.

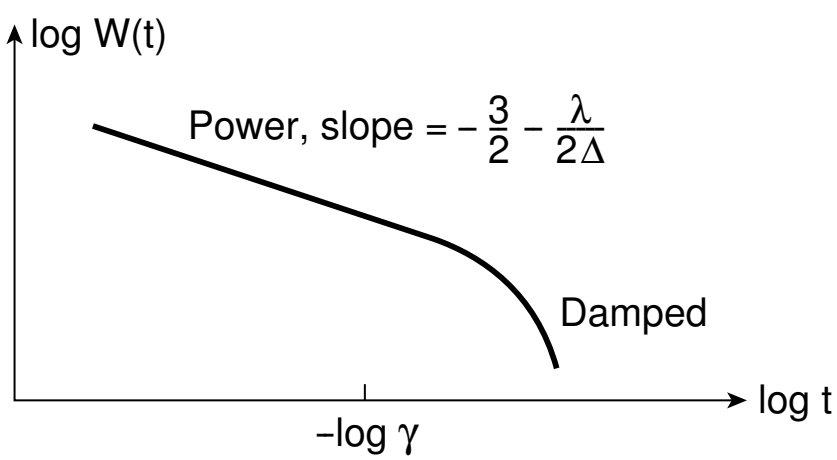

Figure 3. In this figure we sketch the behavior of $W(t)$ in a log$\log$ plot. At intermediate times earlier that $1 / \gamma$ we have scaling behavior with exponent $3 / 2+\lambda / 2 \Delta$, corresponding to a constant negative slope. In the long time limit the curve dips down indicating the cross-over to exponential behavior.

\section{Conclusion}

In this paper we have reviewed the problem of the combined influence of white Gaussian noise of strength $\Delta$ and a linear damping of strength $\gamma$ on a finite-time-singularity of strength $\lambda$. We have for simplicity considered only a single degree of freedom. We find that the first-passagetime distribution $W(t)$ displays a peak about the finite-timesingularity and at intermediate times shorter than $1 / \gamma$ a power law dependence $\propto t^{-\alpha}$, characterized by the scaling exponent $\alpha=3 / 2+\lambda / 2 \Delta$. The exponent is nonuniversal and depends on the ratio between the singularity strength $\lambda$ and the noise strength $\Delta$. In the case where the noise originates from a thermal environment at temperature $T$ we have $\Delta \propto T$ and the scaling exponent depends on the temperature, $\alpha=3 / 2+$ const. $/ T$. At long times later than $1 / \gamma$ the behavior of $W(t)$ crosses over to a an exponential fall-off. To the extent that the character of a finite-time-singularity in the vicinity of threshold can be modelled by a single degree of freedom the present study should hold as regard the influence of noise on the time distribution. We note in particular that in the case of a thermal environment at temperature $T$ the change of the scaling exponent becomes large in the limit of low temperatures as the distribution narrows around the noiseless threshold time. The present study also suggests generalizations to the case of several coupled variable subject to a finite-time-singularity.

\section{References}

[1] M. I. Freidlin and A. D. Wentzel Random Perturbations of Dynamical Systems (Springer, New York, 1998).

[2] R. M. Kerr and A. Brandenburg, Phys. Rev. Lett. 83, 1155 (1999).

[3] M. P. Brenner et al., Phys. Fluids. 9, 1573 (1997).

[4] M. P. Brenner, L. Levitov, and E. O. Budrene, Biophys. J. 74, 1677 (1998).

[5] I. Cohen, M. Brenner, J. Eggers, and S. R. Nagel, Phys. Rev. Lett. 83, 1147 (1999).

[6] J. Eggers, Rev. Mod. Phys 69, 865 (1997).

[7] D. Sornette and J. V. Andersen, Int. J. Mod. Phys. C 13, 171 (2002).

[8] J. Eggers, Phys. Rev. Lett. 89,084502 (2002).

[9] S. Redner, A Guide to First-Passage Processes (Cambridge University Press, Cambridge, 2001).

[10] A. J. Bray, Phys. Rev. E 62, 103 (2000).

[11] J. Farago, Europhys. Lett 52, 379 (2000).

[12] F. Lillo, S. Miccichè, and R. N. Mantegna, (2002), condmat/0203442.

[13] H. C. Fogedby and V. Poutkaradze, Phys. Rev. E 66, 021103 (2002)

[14] H. Risken, The Fokker-Planck Equation (Springer-Verlag, Berlin, 1989).

[15] Mathews and Walker, Mathematical Methods of Physics (Benjamin Press, Menlo Park, 1973). 